\title{
ASSISTENTE PARA AUTOMAÇÃO E MONITORAMENTO DO AMBIENTE: RECONHECIMENTO DE VOZ
}

\author{
André Luiz Amaral Teixeira Costa 1; Esmael Caliman Filho ${ }^{2}$.
}

1 SENAI CIMATEC - Área de Eletrônica Embarcada, Instituto de Inovação em Automação; Salvador/BA; andreamarallcosta@gmail.com.

2 Área | Wyden - Faculdade de Tecnologia e Ciência Area1; Salvador/BA; calimanfilho@gmail.com.

Resumo: Tamanho são os obstáculos que portadores de deficiência física ou com mobilidade reduzida tem que superar em seu dia-a-dia, por isso tornar a sua residência acessível é dar a possibilidade de condição de acesso, qualidade de vida, independência, autonomia e inclusão social. Visando promover uma vida mais independente e restaurando a sua função humana, este artigo propõem um meio tecnológico para facilitar o controle, monitoramento e automação do ambiente de seu convívio. Este trabalho apresenta um sistema de automação, controle e monitoramento utilizando o reconhecimento de voz para interação com o ambiente, baseado no uso do Arduino como controlador, através de uma assistente virtual para interagir com o usuário, atendendo as suas solicitações por controle de voz, otimizando assim a relação dos usuários com os dispositivos eletrônicos de uma residência, sendo uma ferramenta de gestão, que visa otimizar o controle de um ambiente, entregando uma maior segurança para seus utilizadores e facilitando o monitoramento de usuários com necessidades especiais, podendo ser expandido para outros locais, como, hospitais, hotelaria e escritórios. Os testes mostraram os pontos de melhoria e a eficiência do sistema para o objetivo proposto.

Palavras-Chave: Automação Residencial; Monitoramento; Reconhecimento de Voz; Tecnologia Assistiva.

\section{AUTOMATION AND MONITORING ASSISTANT ENVIRONMENT: SPEECH RECOGNITION}

Abstract: Such is the diversity of consumer electronic devices available on the market, that residents have numerous control mechanisms to turn on / off, adapt the settings to the momentary need of the user and everything else that permeates the relation of use of home appliances and portable electronic a residence. This work presents a central system, based on the use of Arduino as the main controller, a virtual assistant to interact with the user, attending requests for voice control, thus optimizing the relationship of users with the electronic devices of a residence. expanded to other environments, such as hospitals, hotels and offices. More than just convenience, the LIS (Life Intelligent System) is an accessibility tool, making life easier for people with some type of disability or difficulty in motor movement. The LIS is a management tool, optimizing costs of an environment, delivering greater security for its users and 
facilitating the monitoring of patients or children. The tests showed the points of improvement and the efficiency of the system for the proposed objective.

Keywords: Automation; Monitoring; Voice recognition; Assistive technology

\section{INTRODUÇÃO}

As pessoas portadoras de deficiências ou com necessidades especiais enfrentam diariamente dificuldades em seus ambientes de convívio. Isto porque na maioria dos casos não conseguem se adaptar com a necessidade da realização de atividades do cotidiano que envolve a utilização da habilidade motora, percepção, flexibilidade, mobilidade, etc. Segundo Comitê de Ajudas Técnicas (2009),"Tecnologia Assistiva é uma área do conhecimento, de característica interdisciplinar, que engloba produtos, recursos, metodologias, estratégias, práticas e serviços que objetivam promover a funcionalidade, relacionada à atividade e participação de pessoas com deficiência, incapacidades ou mobilidade reduzida, visando sua autonomia, independência, qualidade de vida e inclusão social".

Sabe-se que a automação está presente na maioria dos ambientes industriais, garantindo a segurança dos processos, a qualidade dos produtos e a eficiência das indústrias, mas ao longo do tempo, a automação migrou para outros setores da economia, alcançando assim as residências, sendo desenvolvida uma nova área de estudo da automação, chamada domótica. A domótica é uma nova tecnologia que consiste em um sistema integrado capaz de controlar todos os ambientes de uma residência através de um só equipamento, incluindo temperatura, luminosidade, som, segurança, entre outros (BOLZANI, 2004). Diferentemente das industrias, a automação residencial tem como principais objetivos o aumento do conforto, acessibilidade de pessoas com necessidades especiais e segurança. Dados divulgados pela SEBRAE (2016) apontam que o mercado global de automação tem projeção de crescimento anual de 11,36\% entre 2014 e 2020. Prudente (2011, p.1) defende que "A automação predial e residencial, em poucas palavras, pretende identificar todas aquelas tecnologias que permitem tornar automática uma série de operações no interior de um prédio ou habitação".

A automação residencial ainda não foi tão difundida no Brasil, muito se deve por ser uma tecnologia nova, consequentemente de alto custo. Portanto, expandindo o uso da tecnologia assistiva para o controle, monitoramento e a automação, o objetivo deste projeto visa utilizar uma assistente virtual (Life Intelligent Service) gerida pela plataforma microcontroladora Arduino, acessível economicamente, para a automatização do ambiente por reconhecimento de voz, otimizando e facilitando a interação de uma pessoa portadora de qualquer necessidade possível com o ambiente de seu convívio.

Além da introdução, a Seção 2 mostra a metodologia e os procedimentos utilizados na automação, controle e monitoramento de um ambiente residencial por comando de voz. Na Seção 3 são apresentados as discussões e resultados obtidos através da automação. Por fim, na Seção 4, é realizada a conclusão da tecnologia implantada.

\section{METODOLOGIA}


Para desenvolver qualquer tipo de automação são necessários diversos equipamentos que tenham a incumbência de interagir com o ambiente ou sistema, neste caso podendo ser sensores, controladores, relés ou atuadores. Deste modo, para integrar todos esses equipamentos se faz necessário o uso de um controlador para a realização da automação. Sendo assim, o projeto consiste em implantar a automação residencial aplicando o conceito de tecnologia assistiva. Visando a escalabilidade do projeto, foi utilizado o microcontrolador Arduino (2017) como principal controlador do sistema, embarcando diversos periféricos para obter o melhor resultado possível. Para realizar a interação gráfica com o usuário foi utilizado o computador sigle-board Raspberry $\mathrm{Pi}$, que tem o dever de realizar a projeção das imagens interativas em forma de holograma.

O Arduino é uma plataforma eletrônica open source composta por um microcontrolador Atmel AVR que visa de forma fácil e econômica a criação de dispositivos inteligentes capazes de interagir com o ambiente. Ao longo dos anos, o Arduino vem sido cérebro de diversos projetos, desde objetos comuns e até de instrumentos científicos complexos. Composto de uma arquitetura que apresenta 23 pinos de entrada e saída, sendo 14 pinos digitais e 6 analógicos, ele conta com $32 \mathrm{~KB}$ de memória flash onde 512 Bytes são exclusivos e utilizados para o bootloader, $2 \mathrm{~KB}$ de RAM e $1 \mathrm{~KB}$ de EEPROM. A placa pode ser conectada a um computador via USB e programada pela sua IDE, Integrated Development Environment ou Ambiente de Desenvolvimento Integrado, que utiliza uma linguagem de programação com origens em $\mathrm{C} / \mathrm{C}++$.

Quando utilizado, o Arduino normalmente é a plataforma que processa e controla um sistema desejável, como explanado, para este sistema ser um controlador ou gerenciador de um ambiente ele deve conter periféricos que interajam com 0 recinto, no qual neste projeto foram utilizados os sensores, atuadores e shields. Sensor é um dispositivo que faz a detecção e responde com eficiência algumas entradas provenientes de um ambiente físico, como luz, temperatura, movimento, umidade ou qualquer outra variável detectável. Os atuadores são dispositivos que realizam um comando recebido de outro dispositivo com base em um sinal de entrada ou um critério pré-estabelecido. Shields e módulos são placas que tem como função expandir as funcionalidades do Arduino, os circuitos contidos nos mesmos contém uma eletrônica que adiciona funções que a placa principal não possui. Para realizar este projeto utilizou-se 2 Arduinos, divididos entre mestre e slave, um Arduino é responsável por todo o processamento e controle do sistema, enquanto o outro age somente quando é atuado pelo controlador mestre, tal divisão foi necessária por razões de incompatibilidade das bibliotecas dos distintos periféricos. Para a realização deste projeto foi utilizado os seguintes componentes integrados com o Arduino:

- Sensor Light-Dependent Resistor

- $\quad$ Shield EasyVR;

- Shield Mp3 Player Spark Fun;

- Sensor Termistor NTC 10D-9;
- Shield GSM/GPRS;

- Módulo RTC DS1302;

- Sensor de Chama P22;

- Sensor de Gás e Fumaça MQ-2.

\section{RESULTADOS E DISCUSSÃO}


Como resultado da tecnologia aplicada, tem-se respostas assertivas quando se compara este modelo a outras assistentes que hoje existem no mercado, como Google Home, Amazon ou HomeKit. Foi realizado uma pesquisa comparativa da assistente virtual da Google com a assistente deste projeto LIS e foram identificados pontos que fazem uma diferença notória para o usuário no sentido de custo, disponibilidade, funcionalidade e interação gráfica.

\subsection{Custo e Disponibilidade}

O mercado de internet das coisas para a casa tende a crescer muito, mas ainda é pequeno. Investir em uma smart home no Brasil é caro e requer muita pesquisa em sites estrangeiros. Desta forma a assistente LIS tende a ser mais acessível e economicamente viável ao usuário em comparação ao Google Home, tal comparação pode ser visto na Tabela 1 e Tabela 2. Visto que, a tecnologia da Google é totalmente dependente da internet por ser uma IOT (internet of things), ou seja, para um usuário controlar uma televisão, som, ar condicionado ou lâmpadas existe a necessidade de todos esses aparelhos serem smart, que atualmente, no Brasil, não é acessível e nem economicamente viável, assim o valor da sua automação é escalável com os aparelhos smart. Além disso, para a assistente da Google reproduzir algumas funções é necessário possuir assinatura em plataformas externas, como é o caso das músicas, já para ocorrer a integração com a televisão é necessário a compra de um Chromecast. Por fim, além desses pontos citados para fazer o uso da assistente, também é necessário um smartphone para realizar o download e configuração dos aplicativos, tanto da Google Home como os externos para integração. Com base nesses pontos e nas pesquisas realizadas, é notório que para o seu funcionamento, - Google Home utiliza o smartphone como processador e controlador captando os comandos por dois microfones e enviando a saída desejada via rede de internet, desta forma funcionando basicamente como uma caixa com microfone e um alto-falante enviando dados para um celular conectado à internet, a fim de realizar a operação desejada.

Tabela 1 - Cotação de valores dos materiais para o desenvolvimento de LIS

\begin{tabular}{|c|c|c|}
\hline Dispositivos & Quantidade & Valor \\
\hline Arduino & 2 & $\mathrm{R} \$ 25,00$ \\
\hline Shield Easy Vr & 1 & $\mathrm{R} \$ 250,00$ \\
\hline Shield GSM/GPRS & 1 & $\mathrm{R} \$ 50,00$ \\
\hline Shield Mp3 Player & 1 & $\mathrm{R} \$ 24,00$ \\
\hline Modulo RTC & 1 & $\mathrm{R} \$ 4^{2}, 00$ \\
\hline Sensor NTC & 1 & $\mathrm{R} \$ 1_{1,00}$ \\
\hline Sensor de Chama & 1 & $\mathrm{R} \$ 7,00$ \\
\hline Sensor LDR & 1 & $\mathrm{RS} 1,00$ \\
\hline Sensor de Gás & 1 & $R \$ 6,00$ \\
\hline RaspBerry PI-B & 1 & $\mathrm{R} \$ 130,00$ \\
\hline Monitor $18^{\prime}$ & 1 & $\mathrm{R} \$ 250,00$ \\
\hline LEDS & 3 & $\mathrm{R} \$ 1,00$ \\
\hline LED Emissor IR & 2 & $\mathrm{R} \$ 1,00$ \\
\hline Lampada IR & 1 & $\mathrm{R} \$ 30,00$ \\
\hline \multicolumn{2}{|c|}{ Valor Total: } & RŞ 780,00 \\
\hline
\end{tabular}

Fonte: elaborado pelo autor 
Tabela 2 - Cotação de valores dos materiais básicos para utilização do Google Home

\begin{tabular}{|c|c|c|c|}
\hline Dispositivos & Quantidade & Mensal & Valor \\
\hline Spotify & 1 & Sim & R\$ 15,00 \\
\hline Philips Hue & 1 & Não & R\$ 360,00 \\
\hline Google Home & 1 & Não & R\$ 550,00 \\
\hline Google ChromeCast & 1 & Não & R\$ 200,00 \\
\hline \multicolumn{3}{|c|}{ Valor Total: } & R\$ 1110,00 +15,00 p/mês \\
\hline
\end{tabular}

Fonte: elaborado pelo autor

\subsection{Funcionalidade}

Devido à falta de sensores, módulos e atuadores, é notório a ausência de integração da assistente Google com o ambiente físico, desta forma a assistente da fabricante destacada apresenta poucas funcionalidades interativas com o ambiente aplicado, um exemplo disso é que quando o usuário solicita a temperatura, ela informa a temperatura da cidade e não do ambiente em que está, isso se deve pelo fato dela colher essas informações da internet e não de um sensor ou outro componente eletrônico. Por esses motivos, LIS conta com diversos módulos, sensores e atuadores citados neste artigo, apresentando diversas funcionalidades de integração e automatização do ambiente em que está instalada, disponibilizando para o usuário mais segurança e sigilo das informações, integração com os componentes controláveis por infravermelho e trazendo comodidade ao usuário em questão.

\subsection{Interação Gráfica}

Contendo apenas na superfície superior uma tela sensível ao toque que permite o controle do volume da assistente, como pode ser visto na Figura 1, o Google Home chega ao mercado com poucas interatividades além do reconhecimento de voz, no quesito de interação gráfica, o gadget contém apenas $L E D s$ que identificam se o comando foi entendido ou não pela assistente. Diferentemente da assistente da Google, LIS aplica o conceito de holograma e projeção, contendo interações gráficas bastantes significativas e interativas voltadas aos usuários, além dos LEDs que identificam o reconhecimento do comando, a assistente apresentada contém um holograma que interage com o usuário representando por animações os comandos pronunciados, podendo ser visto na Figura 3.

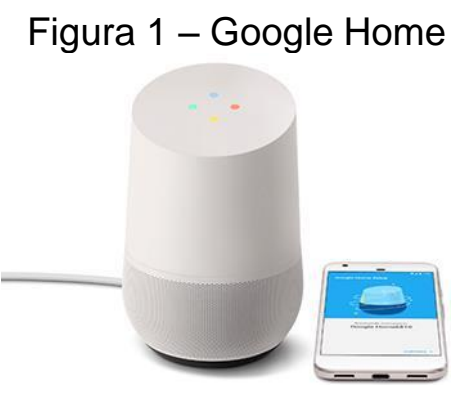

Fonte: Elise (2017) 


\section{Características}

Os benefícios encontrados na utilização da assistente LIS para o monitoramento, automatização, segurança e gestão de um ambiente de convívio de pessoas com deficiências ou mobilidades reduzidas que devem ser destacados são:

- Automatização do ambiente em que está instalada;

- Segurança no controle e monitoramento de temperatura, fumaça e fogo utilizando alarmes sonoros ou silenciosos via sms ou chamadas;

- Sistema multimídia, com músicas, livros, histórias e piadas narradas;

- Plataforma para realização de chamadas e envio de SMS por comando de voz;

- Interface Holográfica animada de acordo com o comando realizado pelo usuário;

- Aplicação independente de internet, facilitando o uso da assistente em qualquer ambiente instalado;

- Código aberto, evitando custo de licença de software.

\subsection{Resultados}

\subsubsection{Testando os Serviços Fornecidos Pela Assistente Virtual}

Para testar o resultado do funcionamento da tecnologia apresentada neste artigo, foi disponibilizado à um deficiente físico, que não tem os dois braços, a utilização da assistente em um ambiente de sua escolha, que no caso, o usuário preferiu que a implantação fosse realizada em seu quarto. Sabendo de sua experiência com a assistente da Google, foi realizado uma série de perguntas ao mesmo, a fim de ser realizado um comparativo satisfatório das duas assistentes discutidas na Seção 3, desta forma, foi constatado através de um questionário que LIS é mais efetivamente funcional, interativa e obteve um custo benefício melhor quando comparado à assistente da companhia Google.

Uma vez instalada em seu aposento, LIS obteve um funcionamento esperado e satisfatório, os motivos ponderados pelo o usuário, são as diversas funções de controle, integração e automatização que a assistente realiza, que segundo ele difere do objetivo da Google Home, pois a assistente da Google era utilizada apenas com finalidade de consulta à internet via comando de voz. Por falta de recursos integrativos da assistente da companhia com o ambiente físico, outro motivo diferencial alegado foi o custo benefício oferecido por LIS.

Foi detectado pelo desfrutador dois pontos necessários de alterações para melhor operação da assistente, um desses pontos foi o ajuste de sensibilidade do sensor de fumaça, pois o usuário é consumidor do cigarro de tabaco, desta forma a assistente enviava notificações via SMS e realizava chamadas sem necessidades, 
alegando fumaça em seu ambiente. Desta forma, foi regulado a sensibilidade no sensor e sanado o transtorno. Outro problema destacado foi a dificuldade que a assistente tinha de entender comandos uma vez que reproduzia áudios na sua plataforma, tal problema se dá pelo motivo do microfone do shield Easy Vr ser do tipo eletreto, problema também constatado na assistente da Google e da Amazon. Sabendo-se disto foi realizada uma adaptação do microfone tipo cardioide e implantado no sistema, sendo assim o microfone captou menos ruídos externo, solucionando o problema.

É possível visualizar na Figura 2 todos os comandos aceitos por LIS e utilizados pelo usuário destacado, pode-se perceber que tais funcionalidades aplicam-se diretamente em seu dia-a-dia por apresentar funções que visam facilitar a sua integração com os eletroeletrônicos e o ambiente de seu convívio.

Figura 2 - Comandos reconhecidos pela Assistente LIS

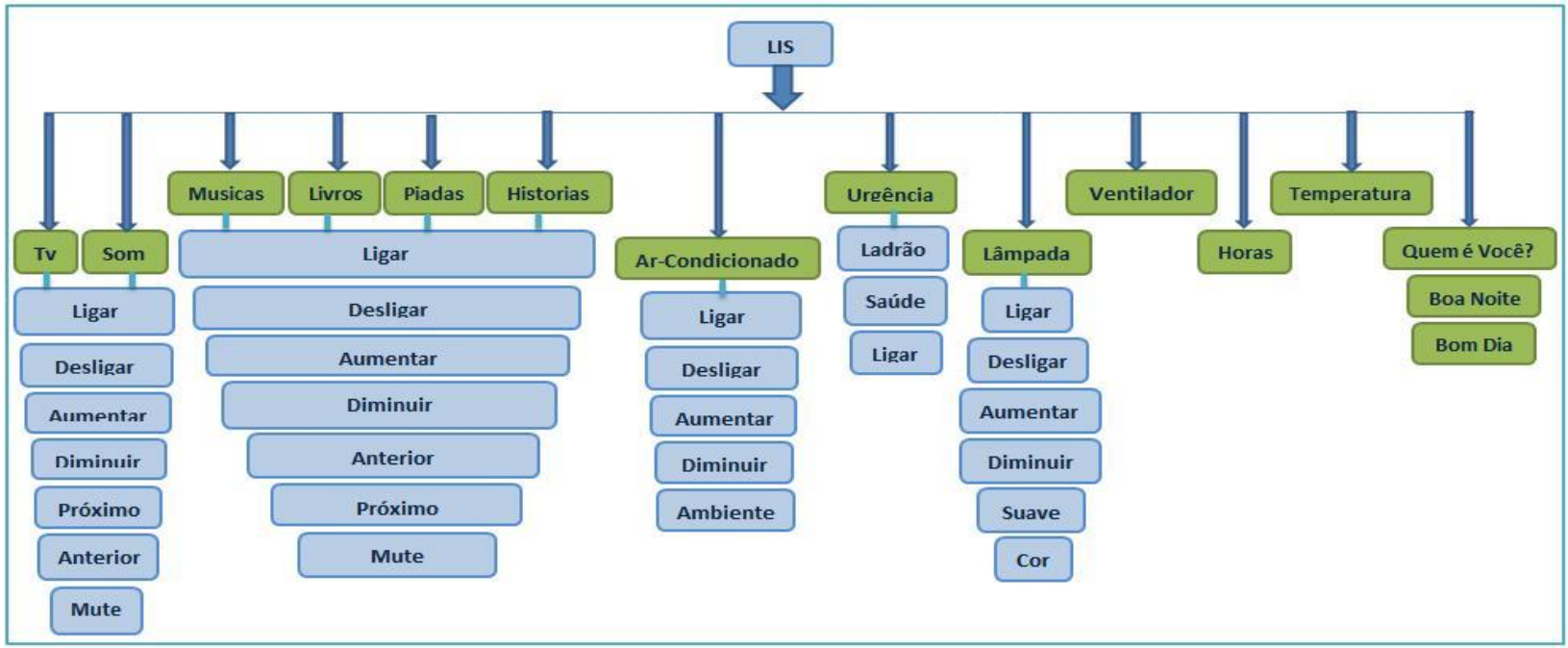

Fonte: elaborado pelo autor

Figura 3 - Comando Ligar Ar-Condicionado e Histórias Contadas do Produto Final
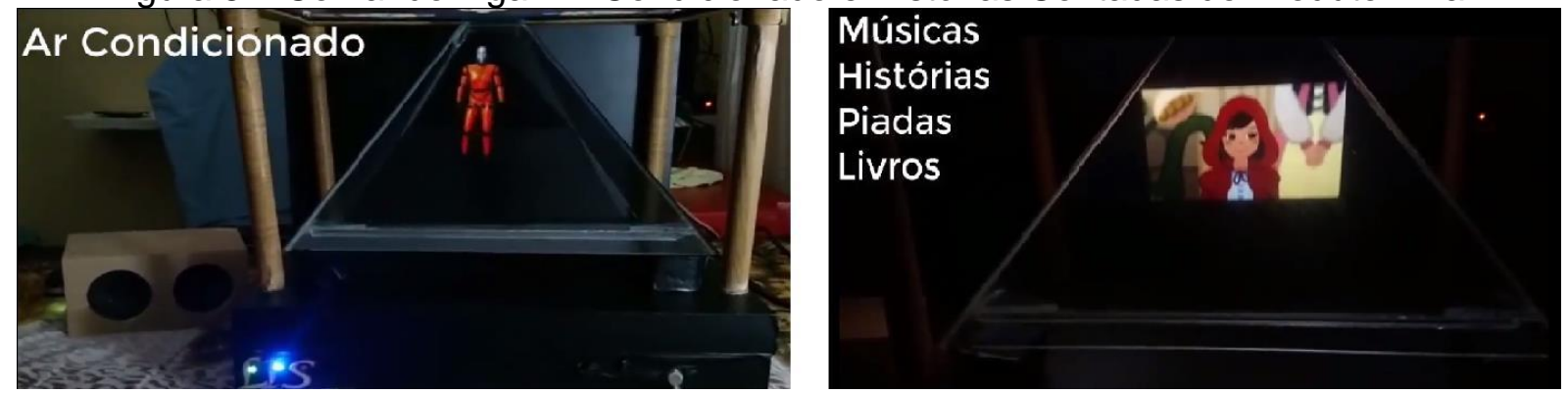

Fonte: elaborado pelo autor

\section{Considerações Finais}

Este trabalho abordou uma solução economicamente viável e funcional para alguns problemas presentes nas atividades do dia-a-dia de pessoas portadoras de 
deficiência física ou com mobilidade reduzida, testando e comprovando a ineficiência das tecnologias que hoje existem com finalidades similares à solução apresentada. Propôs-se aqui um dispositivo diferente, onde o foco é dar ao usuário a restauração da sua função humana, proporcionando uma vida independente e produtiva, utilizando a plataforma microcontroladora Arduino, alguns componentes eletrônicos compatíveis com o hardware e o single-board RaspBery PI.

O Arduino por conter diversos componentes eletrônicos compatíveis com sua arquitetura, demonstrou uma capacidade de escalabilidade muito importante para o aumento da funcionalidade da aplicação proposta, garantindo o uso da assistente para diversas funções que impactam diretamente na vida das pessoas com necessidade especiais. Em conjunto com o Arduino, foi utilizado o RaspBerry PI, que é responsável por fazer toda a parte da integração holográfica da tecnologia assistiva com o usuário. A utilização do single-board com o microcontrolador possibilitou um ótimo desempenho e diversas funcionalidades que hoje estão em carência no mercado voltado a este tipo de tecnologia. Ademais, a automatização, o controle e monitoramento do ambiente nos cenários construídos aqui com as duas plataformas, trouxeram evidências concretas de ser uma solução eficiente para integrar um ambiente utilizado por pessoas portadoras de deficiência física ou motora. Por fim, vislumbra-se a capacidade de expansão da assistente virtual apresentada, com a possibilidade de abranger mais funções para automação, controle, monitoramento e integração, além de estender a sua capacidade de reconhecimento de comandos por voz com a utilização de um terceiro software já existente, compatível com o singleboard RaspBerry PI, conhecido hoje como Google Assistente, presente na maioria dos smartphones.

\section{REFERÊNCIAS}

ARDUINO. Arduino. 2017. Disponível em: <https://www.arduino.cc/>. Acesso em: 18 outubro 2018.

COMITE DE AJUDAS TECNICAS. Tecnologia Assistiva. Brasília: CORDE, 2009. Presidência da República - Secretaria Especial dos Direitos Humanos - Subsecretaria Nacional de Promoção dos Direitos da Pessoa com Deficiência.

BOLZANI, C. A. M. Residências inteligentes: domótica, redes domésticas, automação residencial. São Paulo: Livraria da Física, 2004.

SEBRAE. Automação Residencial: demanda na Construção Civil. 2016. Disponível em:<http://www.aureside.org.br/noticias/automacao-residencial--demanda-naconstrucao-civil>. Acesso em: 16 outubro 2018.

PRUDENTE, Francesco. Automação predial e residencial: Uma introdução. Rio de Janeiro:LTC, 2011. ELISE. Introducing Google Home. 2017. Disponível em: <https://support.google.com/googlehome/answer/7029281?hl=en>. Acesso em: 06 novembro 2017. 